A N N A L E S Annales de Bretagne et des Pays de l'Ouest

\title{
Les syndicats ouvriers des filles de la conserve de poisson en Bretagne 1905-1914
}

Jean-Christophe Fichou

\section{OpenEdition}

Édition électronique

URL : http://journals.openedition.org/abpo/1772

DOI : $10.4000 / a b p o .1772$

ISBN : 978-2-7535-1518-5

ISSN : 2108-6443

Éditeur

Presses universitaires de Rennes

Édition imprimée

Date de publication : 10 juillet 2010

Pagination : 85-100

ISBN : 978-2-7535-1214-6

ISSN : 0399-0826

\section{Référence électronique}

Jean-Christophe Fichou, «Les syndicats ouvriers des filles de la conserve de poisson en Bretagne

1905-1914», Annales de Bretagne et des Pays de l'Ouest [En ligne], 117-2 | 2010, mis en ligne le 10 juillet 2012, consulté le 02 mai 2019. URL : http://journals.openedition.org/abpo/1772 ; DOI : 10.4000/ abpo. 1772 


\title{
Les syndicats ouvriers des filles de la conserve de poisson en Bretagne 1905-1914
}

\author{
Jean-Christophe FICHOU \\ Chargé de cours à l'université de Bretagne Occidentale - Brest \\ Chercheur-membre de Techniques et Culture, CNRS-MNHN \\ Professeur de géographie au lycée de Kerichen - Brest
}

\begin{abstract}
"Quand le capital s'empara de la machine, son cri fut : du travail de femmes, du travail d'enfants [...]. Le système capitaliste développe aussi les moyens de tirer plus de travail du salarié, [...], [en] augment[ant] en apparence le nombre des travailleurs employés en remplaçant une force supérieure et plus chère par plusieurs forces inférieures et à bon marché, l'homme par la femme, l'adulte par l'adolescent et l'enfant ${ }^{1}$. "
\end{abstract}

En 1880, la France est le premier, et l'unique, pays producteur au monde de conserves de sardines à l'huile, distribuées sur la planète entière. Plus de 160 usines échelonnées de Trébeurden au bassin d'Arcachon préparent les poissons bleus, débarqués par 15000 à 20000 pêcheurs et travaillés par autant d'ouvrières. Soumises à l'arbitraire d'un patronat très dur, aux incertitudes de la pêche et gagnant des salaires de misère, ces femmes issues pour la plupart des campagnes environnantes sont totalement inorganisées et vivent dans l'angoisse du lendemain ${ }^{2}$. Les premières lois en leur faveur voient le jour : limitation de la durée quotidienne de travail (1892), " loi des chaises " (1900), libre disposition du salaire par elle-même de la femme mariée (1907)... Pour tenter d'obtenir l'application de ces droits élémentaires et le versement de salaires décents, les " filles de la conserve" commencent à prendre part aux mouvements revendicatifs, bloquent leurs usines et forment les premiers syndicats féminins; pourtant leur audace n'est guère payante car, d'une part, les milieux patronaux ne veulent pas s'en laisser conter et, d'autre part, les organisations ouvrières, masculines, ne sont pas décidées à reconnaître le travail féminin.

1. MARX, Karl, Le Capital, chapitre XXV, Paris, Garnier-Flammarion, 1969, p. 463.

2. Goldmann, Annie, Les Combats des femmes, $X X^{e}$ siècle, Paris/Florence, Casterman/ Giunti, 1996, p. 11. 


\section{Les femmes des conserveries}

L'industrie de la conserverie de poisson, et en particulier de la sardine, demande des manipulations nombreuses qu'il est impossible de mécaniser. Pour réduire les coûts de fabrication, les chefs d'entreprise ont recours massivement à la main-d'œuvre féminine, une des caractéristiques principales de l'évolution industrielle du XIX ${ }^{\mathrm{e}}$ siècle et qui n'est pas spécifique aux conserveries. Mais le phénomène est particulièrement remarquable sur le littoral atlantique, dans des départements par ailleurs faiblement industrialisés. Déjà les statistiques de l'époque le prouvent indubitablement : en 1861, dans le Morbihan, les femmes représentent 68 \% des effectifs salariés des conserveries, et même 89 \% si l'on tient compte des filles mineures ${ }^{3}$. Trente ans plus tard, en 1893, dans le département du Finistère, les femmes et les enfants représentent $77 \%$ des effectifs totaux des conserveries ${ }^{4}$. En 1896, elles forment environ $80 \%$ du personnel des usines sablaises ${ }^{5}$. À la même date, et pour l'ensemble du pays, la part du travail féminin s'établit, sans trop de précision, entre 24 et $35 \%^{6}$, ce qui signifie que cette industrie agro-alimentaire est très féminisée. Le nombre des salariées varie énormément au cours de l'année, en fonction des approvisionnements : en hiver les usines sont vides et elles se remplissent avec l'arrivée des premières sardines. L'effectif évolue aussi énormément selon la taille de la fabrique, d'une dizaine d'ouvrières à plus de 450 pour les plus grandes usines, et cela dès les débuts de l'industrie. En revanche, ces femmes n'ont jamais été considérées par les hommes comme une menace, une concurrence, car ceux-ci n'ont jamais exercé les tâches dévolues aux filles. Le seul personnel masculin des fabriques est constitué par les soudeurs, les mécaniciens d'usine et quelques employés dont les salaires sont nettement supérieurs.

Dans ces usines, le travail n'est pas régulier : on ne sait jamais quand il commence ou quand il finit dans l'année. À plusieurs journées chômées succèdent de longues journées de travail de 16 à 18 heures. On travaille très tôt le matin ou tard dans la nuit. Toutes les filles se plaignent de l'attente, des fatigues du travail nocturne, des heures supplémentaires non rémunérées ${ }^{7}$. Dans tous les cas, la sardine commande. Les conserveurs ont donc besoin d'une population ouvrière peu rémunérée et qui se satisfait de l'instabilité des conditions de travail. Et qui répond le mieux à ces critères, sinon des jeunes filles que les tâches ménagères, conjugales et maternelles, n'appellent pas dans leurs foyers à heures fixes? Le salaire de misère versé constitue un complément indispensable pour les femmes des marins, et surtout leurs filles, bien que ces dernières préfèrent trouver un emploi dans les usines de fabrication des boîtes ou des filets où le travail est mieux

3. Arch. dép. du Morbihan, 5 M 171, Statistiques industrielles 1861.

4. Annuaire statistique, Finistère, 1893.

5. REgOURD, Florence, La Vendée ouvrière. Grèves et ouvriers vendéens de 1840 à 1940, Les Sables-d'Olonne, Le Cercle d'Or, 1981, p. 91

6. BRISSON, Pierre, Histoire du travail et des travailleurs. Paris, Delagrave, 1905, p. 434.

7. Le Journal des Sables, juin 1914. 
rémunéré, plus stable et jugé plus propre, ou dans les usines d'iode où les horaires sont fixes. Pour compléter leurs effectifs, les usiniers font appel très tôt à des filles de la campagne avoisinante. Comme dans la plupart des branches industrielles d'alors, la majorité des ouvrières continuent leurs activités professionnelles après le mariage car, unies à des pêcheurs aux revenus réduits et aléatoires, il leur faut apporter un salaire d'appoint pour faire vivre la famille ${ }^{8}$.

Les fabricants, dans leur grande majorité, n'ont que très peu de considération pour leur personnel féminin, souvent jeune, mis au travail avant les 13 ans réglementaires, et déraciné de l'arrière-pays campagnard. Ainsi, les ouvrières douarnenistes attendent le retour des chaloupes sur un petit champ aux abords de l'Abri du marin «Eh bien, de Penanros [conserveur local] passait et leur disait : N'écrasez pas trop l'herbe, parce que vous en aurez peut-être besoin, les femmes! Vous serez peut-être obligées de la brouter ${ }^{9}$. "Cette main-d'œuvre souvent jugée délurée est crainte, sinon méprisée, par la bonne société locale. D'ailleurs, les plaintes sont nombreuses pour dénoncer l'attitude douteuse de ces jeunes dévergondées. En 1906, l'abbé Le Gallen, en poste à Belle-Île, vilipende cette population d'ouvrières venues pour la saison des sardines de l'intérieur des terres morbihannaises, et parfois même de Concarneau ou de Douarnenez : "Ces ouvrières ne sont en général pas des rosières et les réunions du soir près des établissements sont une cause de démoralisation pour les jeunes gens du pays ${ }^{10}$."

Cette main-d'œuvre saisonnière devient de moins en moins volante, elle se fixe peu à peu et elle fonde des foyers sur place. Cependant, les filles de friteries restent très peu rémunérées sous prétexte qu'elles " dépensent moins que l'homme et qu'elles font moins de travail ${ }^{11}$ ". Même quand elles produisent autant, et parfois plus, que les hommes, comme dans les filatures et les fabriques de vêtements par exemple, elles reçoivent un salaire toujours moindre. Mais, comme le soulignent à chaque occasion les usiniers aux prises avec "l'âpre concurrence " nationale et internationale, comment résisteraient-ils à la tentation de payer une ouvrière 1 franc ou 1,50 franc, alors que, pour le même travail, ils devraient donner au moins le double à un homme? Les salaires sont si faibles en Bretagne que les conserveurs français, découvrant le Portugal après 1881, s'étonnent que la main-d'œuvre portugaise soit autant rémunérée que celle de France. Le fabricant Camille Ogereau est à Cezimbra en 1886 pour chercher un site d'implantation d'usine, et il constate que le salaires versé aux ouvrières est

8. Burdy, Jean-Paul, Dubesset, Mathilde, ZAnCARInI-Fournel, Michelle, " Rôle, travaux et métiers de femmes dans une ville industrielle : Saint-Étienne ", dans Le Mouvement Social, $\mathrm{n}^{\circ} 140,1987$, p. $27-53$.

9. MARTIN, Anne-Denes, Les Ouvrières de la mer. Histoire des sardinières du littoral breton, Paris, L'Harmattan, 1994, p. 42.

10. LE GALLEN, Léandre, Belle-Île, histoire politique, religieuse et militaire. Marine, pêche, agriculture, Vannes, Lafolye, 1906, p. 434-435.

11. Arch. dép. du Finistère, 4 M 209, Douarnenez, le 23 mai 1881, Wenceslas Chancerelle. 
sensiblement le même qu'au Croisic, de 10 à 12 centimes de l'heure ${ }^{12}$. Ce salaire ne progresse que très lentement et de plus en plus de voix s'élèvent, comme celles des députés Georges Le Bail ou Albert de Mun, pour dénoncer l'attitude des usiniers qui ne veulent rien entendre et se contentent de verser le minimum, des salaires de misère, les plus faibles versés dans toute la France ${ }^{13}$. Les hausses, accordées avec parcimonie, sont toujours faibles et obtenues de haute lutte. L'accroissement des salaires est très lent : de moins de 1 franc par jour en 1850 on passe à 2 francs en $1914^{14}$ ! Et si le salaire " a fait une énorme ascension au cours du XIX siècle, ce n'est point à dire qu'il est arrivé très haut. Cela vient à dire seulement qu'il est parti de très bas ${ }^{15}$ ".

Malgré la maigreur des salaires versés, " ce taux misérable ", les ouvrières nous sont toujours décrites par les observateurs comme des femmes heureuses de leur sort, chantant merveilleusement dans leurs usines, surchauffées l'été ou glacées l'hiver :

" Dans cette atmosphère empuantie, vivaient gaies, insoucieuses, ne semblant s'apercevoir de rien, une cinquantaine de femmes, de jeunes filles, dont les éclats de rire bondissaient incessamment d'un coin de la salle à l'autre, tandis qu'un vacarme continuait, composé de mille tapages divers, ébullition de l'huile bouillante, heurt des boîtes de zinc, tintement métallique, traînements de sabots ${ }^{16} \ldots$ ".

Cette version pour le moins idyllique de la vie en usine est fréquemment reprise par l'ensemble des visiteurs d'un jour. En revanche, les rapports des commissaires de police ou des inspecteurs du travail restent toujours muets sur la réalité mythique d'une ambiance joyeuse et insouciante dans les conserveries. On ne chante pas de bonheur. On chante pour résister au sommeil, pour se donner du courage, et qui sait " si les ouvrières n'ont pas fait l'apprentissage de leur force en chantant à l'unisson? ${ }^{17}$ ". Il est certain que la vie est bien plus difficile que celle décrite par les romanciers et les journalistes de l'époque. La présence aux côtés de leurs mères ou de leurs grandes sœurs d'enfants en bas âge aurait d'ailleurs dû les alerter. "Prenez les enfants ", avait dit William Pitt aux industriels anglais qui se plaignaient du manque de main-d'œuvre et des coûts salariaux jugés prohibitifs; les conserveurs français ont très bien entendu le discours et dès les débuts de cette nouvelle activité industrielle, les enfants sont nombreux dans leurs

12. Cité dans Merlant, Yves, "Les Conserveurs nantais au Portugal en 1886 ", Bulletin de la Société archéologique et Historique de Nantes, $\mathrm{n}^{\circ} 117,1981$, p. 178.

13. Dans les filatures du Nord, d'après les résultats de l'enquête officielle menée en 1891-1893, les salaires féminins sont compris entre 1,25 et 2,75 francs. Dans la couture, les " ouvrières du noyau ", celles qui sont les plus occupées, gagnent un salaire réel de 2,15 à 2,85 francs. Dans la confection, l'ouvrière travaille pour un salaire de 1 à 1,25 franc. Les filles des friteries bretonnes touchent par jour de 0,80 à 1 franc.

14. Voir annexe complète en fin d'article.

15. GIDE, Charles, L'Économie sociale à l'Exposition de 1900, Paris, Sirey, 1901.

16. Toudouze, Gustave, Péri en mer, Paris, Victor Havard, 1890, p. 115.

17. MarTin, Anne-Denes, Les Ouvrières de la mer... op. cit., p. 59. 
fabriques. Le développement rapide de cette industrie sardinière a des besoins très importants en main-d'œuvre saisonnière et bon marché. Dans les douze conserveries des Sables et de Saint-Gilles, on compte en 1875 respectivement plus de 300 et 120 fillettes. Toutes les écoles de la région se vident à l'approche de la saison des sardines comme le constatent les inspecteurs de l'Instruction : elles

" deviennent presque désertes pendant la belle saison. On doit en attribuer la cause à l'inexécution de la loi sur le travail des enfants dans les manufactures. Un grand nombre d'écoliers de l'un et l'autre sexe, dès l'âge de cinq ou six ans, sont employés aux usines de conserves alimentaires à un travail qui se prolonge quelquefois bien tard dans la nuit ${ }^{18}$ ".

Il est vrai que certaines tâches ne demandent pas d'efforts physiques démesurés, si bien que de nombreuses ouvrières sont accompagnées de leurs enfants quand elles se rendent à l'usine. Ce complément de revenu, si maigre soit-il, est souvent apprécié car il s'avère indispensable pour faire vivre les familles des cités sardinières. La présence de ces enfants dans des usines bruyantes pendant de longues heures de travail est cependant dénoncée de plus en plus fréquemment.

"Car voici l'horreur des horreurs : le travail des enfants, la misère des tout petits exploitée par l'industrie productrice de richesse! Et cela dans tous les pays! Jésus avait dit : "laissez-les venir à moi." Les riches disent : "Envoyez-les à l'usine, à l'atelier, dans les endroits les plus sombres et les plus mortels de nos enfers. Les efforts de leurs faibles bras ajouteront quelque chose à notre opulence." [...] Que penser du bruit, [...], des pas innombrables de ces pauvres petits allant à leur tâche de souffrance et de misère exigée par les maudits ${ }^{19}$."

Il ne semble pas que la grandiloquence des textes de Léon Bloy ait ému nos conserveurs qui se séparent de la main-d'œuvre enfantine à regret, contraints et forcés par des textes législatifs qu'ils combattent avec acharnement. Comme la tenue des comptes des fabricants est fondée sur une stricte économie de gestion, pour eux la limitation au plus juste des charges salariales ou sociales reste un dogme, loin d'être fondé d'ailleurs. Dans ces conditions, les salaires sont toujours très bas et les augmentations, si faibles soient-elles, sont toujours obtenues de haute lutte ${ }^{20}$.

\section{Les ouvrières et le syndicalisme}

Si les grèves des pêcheurs et des soudeurs restent relativement connues, celles des ouvrières sardinières sont moins retentissantes. La main-d'œuvre féminine des conserveries n'est pas syndiquée et n'a pas

18. Arch. dép. de Vendée, 10 M 15, Inspection du Travail, 1878. Cité par REGOURD Florence, La Vendée ouvrière..., op. cit., p. 92.

19. Bloy, Léon, Le Sang du pauvre, Ed. Stock, Paris, 1948 (1909), p. 177.

20. DuBoIs, Xavier, La Révolution sardinière : pêcheurs et conserveurs en Bretagne Sud au XIX $X^{e}$ siècle, Rennes, Presses universitaires de Rennes, 2004, p. 295 et suivantes. 
développé une conscience politique très forte, loin de là, mais à leur corps défendant : il faut préciser que ce sont les syndicats eux-mêmes qui s'intéressent peu au travail des femmes et à leurs problèmes. Elles ne restent pas pour autant les bras croisés et participent à la création des associations de défense, comme à Morlaix en 1891 où est créé le premier syndicat de la Manufacture des Tabacs qui compte une majorité d'ouvrières ${ }^{21}$.

Le premier mouvement féminin de protestation retrouvé dans les conserveries se déclare assez paradoxalement sur l'île d'Yeu où personne ne s'attend à des débordements d'ouvrières réputées calmes. Le 27 mai 1889, deux cents filles travaillant dans les trois usines insulaires, Saupiquet, Amieux et Bouvais-Flon, se mettent en grève car les gérants ont décidé de réduire leurs salaires horaires de 5 centimes sous prétexte que la sardine n'est pas suffisante. Cette réaction d'humeur épidermique des salariées est totalement inattendue et laisse perplexe un patronat décontenancé qui préfère ne pas opérer la baisse de salaire envisagée ${ }^{22}$. Cependant, le personnel féminin des conserveries reste, dans son immense majorité, très résigné. Les autorités sont confiantes et ne redoutent aucune flambée de violence, tout du moins jusqu'en 1894, année où la situation s'envenime :

"Les usines de conserves établies sur la côte de l'arrondissement de Lorient ne fonctionnent que pendant la saison de la pêche ou pendant celle de la récolte des légumes. Ce sont en majorité des femmes qui travaillent dans ces "fricasseries" comme on dit ici. Là encore il n'y a pas à craindre de grève, et si par hasard pareille tentative se produisait les directeurs de ces maisons trouveraient facilement un nouveau personnel tout prêt à prendre les places de celui qui refuserait le travail. Aucune connaissance spéciale n'est en effet exigée et depuis la fermeture d'un grand nombre d'usines, il reste malheureusement un grand nombre de mères de famille qui attendent les places pour pouvoir à leur tour reprendre le travail ${ }^{23}$."

Les mouvements de ces filles de friture sont encore plus impulsifs et incontrôlés que ceux des pêcheurs, pourtant déjà peu disciplinés, et plus encore des ferblantiers, mais leur ténacité et leur pugnacité n'ont rien à leur envier ${ }^{24}$. Les conflits du travail deviennent non seulement un moyen de pression sur le patronat " pour obtenir la satisfaction des revendications, mais aussi un moyen d'expression : ne pas avoir, mais être ${ }^{25}$ ". À Port-Louis, au cours de la grève des pêcheurs de juin 1897, les femmes et les ferblantiers se retrouvent au chômage faute de poissons à préparer. De cette inactivité est peut-être née la discussion; toujours est-il que les femmes cessent

21. MiCHEL, Claude, Esquisse d'une histoire sociale de la Bretagne, Lorient, Institut CGT d'Histoire sociale de la Bretagne, 1993, p. 22.

22. Arch. dép. de Vendée, 10 M 58, la Roche-sur-Yon, le 29 mai 1889, la préfecture.

23. Arch. dép. d'Ille-et-Vilaine, 1 U Parquet, grèves 1892-1896. Lorient, le 20 janvier 1896 , le procureur.

24. Fichou, Jean-Christophe, Les Conserveries de sardines et les conserveurs - littoral atlantique français, 1852-1969, Brest, UBO, mémoire d'Habilitation à Diriger les Recherches, p. 307.

25. JaVILliER, Jean-Claude, Les Conflits du travail, Paris, PUF, 1981, p. 24. 
le travail dès que les pêcheurs reprennent la mer le 15 juin. Les revendications sont, comme toujours, avant tout salariales :

"Les femmes employées dans les usines demandent une augmentation de 0 franc 90 par mille de sardines, ce qui porterait le prix à 1 franc 90 au lieu de un franc [...] L'usine Philippe a déjà reçu une délégation et a accepté, les usines Delory et Penaud auraient refusé mais rien n'est définitif ${ }^{26}$."

La grève est très suivie, ce qui inquiète le commissaire de Port-Louis ; c'est d'autant plus sérieux selon lui " qu'il y a entre elles entente et solidarité, ce qui n'existe pas chez les pêcheurs ${ }^{27}$ ". Après deux jours de grève, les ouvrières retrouvent leurs fabriques, mais après avoir obtenu satisfaction, puisque toutes reprennent le travail avec une augmentation de salaire au mille comprise entre 25 et 50 centimes.

"Les femmes employées au nettoyage des poissons ont réclamé une augmentation de salaire de $20 \%$ sous peine de cesser le travail immédiatement. Les usines ont donné satisfaction et elles seront désormais payées 1,25 du mille au lieu de 1 franc $^{28}$."

Cette victoire ouvrière indiscutable est un motif de crainte avoué par tous les usiniers qui continuent cependant d'offrir des salaires dérisoires, " des salaires de famine, [...] du vol ni plus ni moins ${ }^{29}$ ". Surtout, ils n'envisagent jamais d'augmentation sinon contraints et forcés. Ils mettent en avant, avec une mauvaise foi évidente, les répercussions insurmontables sur le prix de vente des boîtes entraînées par des efforts salariaux déraisonnables. Les petites maisons peu connues sont d'ailleurs les plus ladres et survivent en présentant de " la camelote ». Ces produits évidemment ne " pourront s'écouler qu'à cause de leur bon marché, et on économisera sur la main d'œuvre comme on a économisé sur le produit lui-même ${ }^{30}$ ". Pourtant, quand on regarde de près les coûts de production, on remarque invariablement que les salaires versés ne représentent jamais que 5 à $10 \%$ du prix des conserves de sardines à l'huile, que cette part ne cesse de baisser entre 1860 et 1930, que les salaires sont toujours les plus bas versés dans toute la France ouvrière. Et cet état de fait perdure de longues années : au cours de la grande grève de décembre 1924, à Douarnenez, l'inspecteur divisionnaire du Travail le note sans équivoque :

" Aux cours pratiqués cette année, la main-d'œuvre ne représente pas $6 \%$ du prix mis en œuvre pour les conserves de sardines d'où l'existence d'une marge très large permettant de relever les salaires des ouvriers et des ouvrières occupés à ces fabrications ${ }^{31}$."

26. Arch. dép. du Morbihan, 1 Z 176, Port-Louis, le 14 juin 1897, le commissaire au sous-préfet.

27. Ibid, le 16 juin 1897 , le commissaire au sous-préfet.

28. Le Populaire du 20 juin 1897.

29. Le Réveil du Finistère, le 25 mars 1905.

30. De SellHAc, Léon, La Pêche de la sardine. Paris, Masson et Gauthier-Villars, nd (1903), p. 97.

31. Arch. dép. du Finistère, 10 M 54, Nantes, le 14 décembre 1924, l'inspecteur divisionnaire Gillet. 
Malgré une exploitation éhontée des femmes de friture, l'organisation syndicale de ces ouvrières est très longue à se dessiner et plus encore à se mettre en place. C'est à Douarnenez, de nouveau, que le premier syndicat ouvrier strictement féminin est créé, né de la grève. En février 1905, les filles et les femmes sont descendues dans les rues pour obtenir un salaire non plus au mille de sardines préparées, mais à l'heure afin de faire augmenter des salaires très bas. Le système du mille ne permet pas aux ouvrières de contrôler le nombre exact de sardines travaillées. Elles se plaignent de "l'impossibilité où elles sont de discuter avec le gérant pour établir si le nombre de milles qui leur est attribué n'est pas en dessous de leur travail effectif ${ }^{32}$ ". Bon nombre d'usiniers profitent de ces difficultés de comptage pour minorer les salaires, lesquels dépendent en définitive du seul bon vouloir de la commise, ou contremaîtresse, surveillant les travaux " ayant ses têtes de turcs et ses amies très chères, achetant le poisson et le faisant transformer au mieux des intérêts de la maison, c'est-à-dire aux pires de ceux des ouvrières ${ }^{33}$ ". Les ouvrières douarnenistes sont d'autant plus remontées qu'elles savent que le tarif à l'heure fonctionne déjà à Étel, à Quiberon, à La Turballe, où la main-d'œuvre est plus rare. À la vue des 3000 femmes dans les rues, les fabricants locaux accèdent rapidement à leur demande : le 11 février, Charles Chancerelle est le premier à accepter, suivi le lendemain par la majorité de ses collègues ${ }^{34}$. Les ouvrières, tout à leur joie, décident de pousser plus avant cette expérience de lutte. Elles se doutent bien que leur victoire est loin d'être acceptée de gaieté de cœur par les usiniers pour qui " jamais une femme, jamais un homme ne travaillera aussi vite à l'heure qu'à la pièce, qu'au mille : c'est indiscutable ${ }^{35}$ ". La première réunion préparatoire pour la formation d'une organisation féminine doit se tenir le 25 février à 14 heures dans la salle de Venise mais les patrons, un rien mesquins, qui ont " l'habitude de ne faire la paie qu'à 4 heures ont affiché qu'elle se ferait à 2 heures et ce dans le but de retenir leurs ouvrières pour les empêcher de se rendre à la réunion ${ }^{36}$ ".

Malgré tout, Le Gall, de la Bourse du Travail de Brest, appelle les 150 ouvrières présentes à s'unir pour présenter un front uni et renforcé face aux exigences patronales et s'accorder sur une base pour le moins simple, la revendication d'un "salaire vital ». L'orateur parvient à subjuguer l'assemblée puisqu'à la fin du discours, les salariées présentes élisent un bureau pour les représenter et accomplir les démarches administratives

32. ROPERS, Louis, Exposition de la condition économique et sociale du pêcheur sardinier, Paris, Bonvalot-Jouve, 1906, p. 45.

33. LE Coz, Bruno, La Crise sardinière au pays bigouden maritime 1902-1908, Brest, UBO, mémoire de maîtrise, 1985, p. 42.

34. Le Boulanger, Jean-Michel, Douarnenez de 1800 à nos jours, Rennes, PUR, 2000, p. 220 .

35. La Libre Parole, le 20 juillet 1905. Le journal d'Édouard Drumont prend fait et cause pour le patronat et dénonce le caractère socialiste du syndicat des ouvrières.

36. Arch. dép. du Finistère, 4 M 209, Douarnenez, le 25 février 1905, le commissaire au préfet. 
nécessaires à la création du Syndicat des ouvrières sardinières ${ }^{37}$. Mais, très vite, les premières difficultés apparaissent, et notamment la volonté affichée des usiniers de licencier toutes les ouvrières syndiquées. Pour les observateurs, il est à craindre que "l'ère des difficultés ne soit pas close définitivement et que les patrons ne tentent pas quelques moyens de revenir sur les concessions qu'ils ont dû faire ${ }^{38}$ ". En fait, ce sont les membres du syndicat des marins pêcheurs de Douarnenez qui prennent officieusement la direction, avancent les fonds nécessaires au fonctionnement et se démènent pour recruter de nouvelles adhérentes. Fortes de leur nombre, les ouvrières réclament un contrat définitif, signé par tous les usiniers douarnenistes, entérinant le paiement à l'heure et l'embauche en priorité des ouvrières syndiquées. Le 5 mars 1905, on compte officiellement 359 syndiquées, mais ce chiffre ne cesse de décliner par la suite; Émilie Guéguen et ses consœurs à la tête du comité connaissent des difficultés croissantes pour convaincre les usiniers mais aussi les ouvrières " car beaucoup d'entre elles craignent les représailles du clergé ${ }^{39}$ ». Le Syndicat d'ailleurs finit par disparaître faute de paiement des cotisations. Mais au cours des appels lancés pour obtenir des subsides, les orientations politiques du groupement apparaissent clairement : les ouvrières syndiquées,

" [...] après avoir marché à l'avant-garde des revendications prolétariennes, se voient en butte à toutes les tracasseries patronales [...] Camarades de toutes professions vous apporterez votre obole, vous soutiendrez le syndicat des sardinières qui s'est promis d'émanciper les bretonnes esclaves jusqu'ici de tous les préjugés et de tous les jougs capitalistes ${ }^{40}$ ".

Il semble bien que cette orientation révolutionnaire prononcée du discours ait choqué beaucoup d'ouvrières qui abandonnent rapidement l'association. Il est vrai que l'ensemble de la presse bien-pensante se démène pour rappeler à chaque occasion que la grève n'est jamais choisie naturellement par les filles :

"Rien qu'à Douarnenez pour trente usines, on en compte plus de 1000 fédérées en un syndicat qui obéit aux ordres des gréviculteurs parisiens. Ce syndicat, en 1905, décréta une première fois la grève, parce que les fabricants ne voulaient pas remplacer le travail au mille, qui stimule l'initiative, par le travail à l'heure et qui ne profite [...] qu'aux paresseuses et aux maladroites ${ }^{41}$."

37. Arch. dép. du Finistère, 4 M 210. Émilie Guéguen, secrétaire générale, Marianne Kerloc'h, secrétaire, Françoise Pallud, trésorière, Eulalie Belbeoc'h, Angeline Gonidec, Marie Andro, Anna Guéguen, membres.

38. Ropers, Exposition de la condition..., op. cit., p. 45.

39. Martin, Anne-Denes, Les Ouvrières de la mer..., op. cit., p. 137.

40. Arch. dép. du Finistère, 4 M 210, cité par le commissaire spécial, Quimper, le 30 décembre 1905.

41. LE GofFIC, Charles, "La Crise sardinière ", Revue des Deux Mondes, 15 janvier 1907, cité dans AmIEux, Louis, Documents concernant la crise sardinière. 1903-1912, Nantes, Dugat, 1913, p. 15. 
De plus, il ne faut pas oublier que les femmes mariées ne peuvent adhérer aux syndicats sans l'autorisation de leur époux et qu'elles sont peu disponibles, portant le poids de la double journée de travail ${ }^{42}$. Il faut attendre 1920 pour que cette interdiction soit levée. Au début du Xx siècle les marins, leurs pères, leurs frères ou leurs maris, ne les soutiennent pas forcément et se plaisent au contraire à entretenir le "stéréotype de la gréviste folle, coureuse et virago ${ }^{43}$ ". La presse locale n'est jamais tendre non plus à leur égard et présente les adhérentes comme des célibataires dévoyées sans enfant ${ }^{44}$ et les meneuses sous les traits de mégères excitées, paillardes, vives et emportées; c'est ainsi que les dépeint le Vendéen lors du conflit de 1909 aux Sables, qui met en scène les ouvrières de la cité ${ }^{45}$.

En 1905, Le Gall, après avoir décidé les filles de Douarnenez à créer leur syndicat, tente une expérience similaire à Concarneau. Notre orateur se présente le 13 mai dans cette dernière ville, devant 700 ouvrières rassemblées pour la circonstance, mais il ne peut se faire entendre. Son discours est interrompu à chaque instant par des cris et des huées, la moitié de l'assistance lui étant très hostile, et aucune injure ne lui est épargnée. Bien entendu aucun syndicat d'ouvrières ne voit le jour à Concarneau ${ }^{46}$. Les difficultés sont grandes pour amener ces ouvrières frustes à se regrouper et découvrir l'efficacité annoncée de l'unité syndicale, d'autant plus que les fabricants associent leurs efforts pour réduire considérablement l'audience des ouvrières syndiquées. À Douarnenez, ils organisent dans un premier temps un second syndicat modéré sous la houlette de Louis de Pénanros, célèbre conserveur du cru, puis, dès juillet 1905, ils " donnent le travail au mille » malgré les engagements signés en février. Le patronat de la conserverie approuve l'attitude de leur confrère qui reçoit aussi le soutien de l'Église locale et de l'évêque de Quimper, reprenant à son compte les idées développées dans l'encyclique Rerum novarum par Léon XIII pour contrecarrer l'influence naissante de la CGT ${ }^{47}$. Le journal L'Action est encore plus clair dans son propos et déclare que tout " fut mis en œuvre pour vaincre les ouvrières de leurs si légitimes revendications ${ }^{48}$ " en confiant cette tâche au clergé local : "Les prêtres refusent l'absolution et tous les sacrements aux femmes soupçonnées de vouloir travailler à l'heure ${ }^{49}$. "Ils sont d'ailleurs épaulés dans cette action par le préfet du Finistère, Collignon, dont l'implication dans cette affaire, aux côtés des fabricants, est très mal vue par les pêcheurs et les ouvrières qui boudent le syndicat trop proche du patronat.

42. RatTo, Martine, GauTiER, Andrée, "Les Syndicats féminins libres de l'Isère 1906-1936 ", Clio, 3/1996, sur http://clio.revues.org.

43. Schweitzer, Sylvie, Les Femmes ont toujours travaillé, Paris, Odile Jacob, 2002, p. 127.

44. Ouest-Éclair, 13 février 1906.

45. Arch. dép. de Vendée, 11 M 121, cité dans REGOURD, Florence, La Vendée ouvrière... op. cit., p. 201.

46. Arch. dép. du Finistère, 10 M 34. Concarneau, le 13 mai 1905, le commissaire au préfet.

47. Ibid., Concarneau, le 27 mai 1905, le commissaire.

48. L'Action, le 22 juillet $1905,1^{\text {er }}$ thermidor an 113.

49. Arch. dép. du Finistère, 4 M 210, Douarnenez, le 9 juillet 1905, le commissaire. 
À son tour, le député de la circonscription, Le Bail, intervient promptement pour soutenir les filles et dénoncer la position des fabricants, mais son action est jugée par le commissaire spécial assez futile et pour le moins démagogique :

" Il a fait sommation aux usiniers réfractaires d'avoir à travailler à l'heure et, au cas où ils ne se soumettraient pas, il les assignera devant le Tribunal civil en [demandant] 20000 francs de dommages-intérêts. Ces dispositions permettront très vraisemblablement d'atteindre la fin de la campagne de pêche et l'expiration du contrat à l'heure avant qu'une solution soit intervenue $^{50}$."

Évidemment cette attitude patronale envenime une situation déjà tendue : «Un vent de révolution souffle sur la ville ${ }^{51}$. "Quarante gendarmes et une compagnie du $118^{\mathrm{e}}$ de ligne sont maintenus sur place pour éviter tout débordement. Le 10 juillet, les filles de l'usine Parmentier refusent leur paie calculée au mille et réclament le bénéfice de leurs revendications acceptées cinq mois auparavant. Toutes les usines, sauf quatre, préfèrent alors répondre favorablement aux exigences des ouvrières avant l'embrasement généralisé et la campagne s'achève à peu près calmement.

" Le ciel s'est rasséréné en Bretagne et l'accord s'est fait entre les sardinières et les usiniers sur la question du travail à l'heure qui remplacera le travail au mille, solution toute pacifique d'un conflit qui commençait à prendre mauvaise tournure [...] Ah les braves filles, et si gaies, si rieuses, la tête un peu trop près du bonnet, sans doute, mais le cœur si chaud et l'âme si compatissante ${ }^{52}$."

Mais dès le mois de novembre, la bataille reprend. Le 16 novembre, les fabricants refusent d'accorder des contrats pour la saison future, en fait des arrhes ${ }^{53}$, aux ouvrières membres du bureau, dont "Émilie Guéguen et ses compagnes, d'opinion très avancée ${ }^{54}$ ". Craissac, membre de la CGTsyndicat des peintres, tente d'intercéder en leur faveur mais il avoue son incapacité à régler le conflit. Il déclare que ses "démarches n'avaient pas abouti et qu'ayant largement réfléchi il était d'avis qu'il n'y avait pas lieu de recourir à la grève générale, [...], le syndicat est complètement disloqué ${ }^{55}$ ".

Il est clair que l'échec du mouvement est dû à l'action efficace des patrons de conserveries qui sont dans leur grande majorité des réactionnaires catholiques qui utilisent tous les moyens, en association avec le clergé local, pour conserver leur rang. Les marins pêcheurs syndiqués de

50. Arch. dép. du Finistère, 10 M 37, Quimper, le 21 juillet 1905, le commissaire spécial au préfet.

51. Le Matin, le 20 juillet 1905.

52. Arch. dép. d'Ille-et-Vilaine, 4 Fg 103, Le Petit Journal du 3 août 1905, « Les Sardinières ".

53. Les industriels, pour fixer durant toute la campagne le personnel féminin, versent des arrhes au début de la saison. Ces arrhes sont remboursées par les premières paies et les ouvrières sont du même coup engagées.

54. Arch. dép. du Finistère, 10 M 37, Quimper le 18 novembre 1905, le commissaire spécial au préfet.

55. Ibidem. 
la ville constatent tous les grandes difficultés rencontrées par les ouvrières pour s'organiser. Pour eux, les explications sont simples :

" [...] Il paraît que les calotins veulent démolir leur syndicat [des ouvrières sardinières] et passent des menaces aux faits. Dans une seule usine (Chancerelle), 28 syndiquées ont déjà vu résilier leur engagement. Dans les autres usines la proportion est équivalente $[\ldots]^{56}$. "

L'année suivante, les ouvrières de Saint-Guénolé, qui touchent alors entre 5 et 15 centimes de l'heure pour un "travail qui se poursuit parfois jusqu'à 20 heures sans suspension ", se réunissent pour envisager la grève et la création d'un syndicat :

«Et, malgré les craintes des fabricants, il est à supposer que la suspension de travail ne se produira pas dans ce pays où la femme, encore un peu primitive, a conservé le respect du "maître" et n'oserait, par suite, pas tenter un soulèvement ${ }^{57}$."

Dans les autres ports sardiniers, les mouvements féminins de contestation sont beaucoup plus rares : les ouvrières de Port-Louis manifestent au cours de l'été 1897, nous l'avons évoqué, et des incidents du même genre se déroulent aussi au Croisic mais c'est tout ${ }^{58}$. Il faut attendre les grands affrontements de 1909 et 1910 entre les pêcheurs et les patrons, ou les grèves dures des soudeurs, pour que les associations revendicatives féminines s'organisent de nouveau en passant par la création de syndicats. Peut-être aussi le vote de la loi du 13 juillet 1907, autorisant les femmes mariées à disposer librement de leur salaire ${ }^{59}$, a-t-il encouragé ces dernières à présenter des revendications mieux préparées et plus virulentes, car désormais elles se battent pour un bien dont elles peuvent jouir comme bon leur semble. La CGT commence aussi à prendre plus sérieusement en compte les réclamations de ces femmes et s'inquiète de l'éducation et de la syndicalisation féminines ${ }^{60}$. Le syndicat des ouvrières sardinières de Douarnenez est reconstitué le 28 octobre 1909; le premier mai 1910 est créé celui de Concarneau où Le Gall est revenu pour rappeler de nouveau les bienfaits de l'union ouvrière, cette fois-ci avec plus de succès. Ce dernier syndicat, entièrement féminin, décide la grève le 11 juillet pour obtenir des augmentations de salaire; le 14 juillet les fabricants acceptent ces hausses qu'au demeurant ils trouvent " justifiées ${ }^{61}$ ". Un dernier syndicat d'ouvrières est créé en juillet 1914 au Guilvinec ${ }^{62}$ mais

56. Arch. dép. du Finistère, 4 M 209, Douarnenez, le 28 mars 1905, le secrétaire du Syndicat des marins pêcheurs.

57. Arch. dép. du Finistère, 4 M 210, Quimper, le 28 juin 1906, le commissaire spécial.

58. Cité dans Gesun, Claude, Le Syndicalisme ouvrier en Bretagne jusqu'à la Première Guerre mondiale, Saint-Hippolyte-du-Fort, Espaces écrits, 1990, p. 221.

59. Tiano, André, Les Pratiques publiques d'exclusion depuis la Révolution française, Paris, l'Harmattan, 1999, p. 19.

60. SCHWEITZER, Sylvie, Les Femmes ont toujours travaillé..., op. cit., p. 122.

61. Ouest Éclair, le 15 juillet 1910. Déclaration d'Arthur Benoist, président du syndicat des fabricants de Nantes.

62. GesLin, Claude, Le Syndicalisme en Bretagne..., op. cit., p. 454. 
il n'a, semble-t-il, qu'une durée éphémère, emporté par les tourbillons de la Grande Guerre.

Sur la frange littorale industrielle de la France de l'Ouest, les ouvrières et les ouvriers des conserveries, les pêcheurs aussi, restent des populations relativement isolées face à un patronat organisé dès qu'il s'agit de défendre ses intérêts. Seuls les soudeurs parviennent à créer un syndicat suffisamment puissant pour faire entendre leurs voix mais sans pour autant que leurs revendications aboutissent; par ailleurs, ils n'obtiennent jamais la confiance des autres professions en butte aux ordres des usiniers. Leurs jours sont comptés à brève échéance car ils forment un groupe trop combatif, craint des conserveurs qui s'empressent de les remplacer par des machines dès que la possibilité leur en est offerte. Les rares mouvements de grève dans ce secteur d'activité que l'on note en Bretagne ou en Vendée entre 1880 et 1900, qu'il s'agisse des pêcheurs ou des salariés, n'ont jamais qu'une portée très restreinte et s'apparentent plus à des réactions d'humeur qu'à de véritables mouvements préparés. Il s'agit de la concrétisation d'une animosité épidermique accumulée depuis des années mais sans qu'une entente d'envergure ne voie jamais le jour. Les femmes sont encore trop effacées pour jouer un rôle revendicatif important dans une société où leur place est de toute manière parfaitement fixée. Malgré les efforts des socialistes et des syndicalistes locaux, voire nationaux, les tentatives de création d'organisations professionnelles revendicatives se soldent par autant d'échecs dans un monde syndicaliste masculin. Elles combattent des fabricants organisés qui présentent une ligne de conduite stable tout en apportant des réponses très dilatoires, différentes selon les ports, selon les usines, et en jouant sur les antagonismes des différentes communautés, entre les pêcheurs de thon ou de sardine, entre les ports, entre les Finistériens et les Morbihannais, entre les Bretons et les Vendéens. Malgré toutes ces entraves, malgré leur passivité liée, il est vrai, à leurs conditions d'emploi précaire ${ }^{63}$, les " filles de la conserve " ont réussi à organiser quelques grèves et même un syndicat spécifique. Cependant, si les premiers éléments d'un syndicalisme féminin ouvrier se mettent en place, les résultats sont décevants et éphémères, si bien que les conserveurs parviennent à surmonter la crise en imposant à leur main-d'œuvre des restrictions supplémentaires tout en continuant de verser des salaires dérisoires.

63. BARD, Christine, Les Femmes dans la société française au 20e siècle, Paris, Armand Colin, 2001, p. 25. 


\section{Annexe - Salaires des femmes dans les conserveries françaises}

Ces données ont été collectées à partir de sources très variées. Pour plus de détails, voir : " Les Conserveries de sardines et les Conserveurs - Littoral atlantique français (1852-1969) ", mémoire inédit d'Habilitation à Diriger des Recherches, UBO, Brest, 2006, 956 p.

1847 : 0,60 F par jour à l'usine Soymié d'Étel; 0,80 F à l'usine Rödel d'Erdeven.

1853 : 700 à $1000 \mathrm{~F}$ pour la saison à l'usine Peyron de Doëlan.

1855 : 0,80 à 1,00 F par jour à l'usine Caillo du Croisic.

$1862: 0,80$ à 1,00 F par jour dans les usines de Port-Louis.

1875 : 1,00 F par jour dans les usines de Concarneau

de 1877 à $1889: 1,00 \mathrm{~F}$ par jour dans les usines de Concarneau.

1882 : de 0,60 à $1,00 \mathrm{~F}$ par jour dans le Finistère.

1886 : de 0,15 à $0,20 \mathrm{~F}$ de l'heure à Quiberon et au Palais; 0,10 à Concarneau; 0,30 à Port-Louis; de 0,12 à 0,25 au Croisic.

1889 : 0,20 F de l'heure à l'usine Bouvais-Flon de l'île d'Yeu.

1890 : de 2 à 3 francs par jour à Port-Louis.

1896 : 36 à $40 \mathrm{~F}$ par mois à l'usine Jego de Groix ( $0,10 \mathrm{~F}$ de l'heure).

1897 : 0,10 F de l'heure à Port-Louis; en grève elles obtiennent 5 centimes d'augmentation horaire.

1901 : 0,15 F de l'heure à l'usine Basset de La Rochelle.

1902 : 1,35 à 1,50 F par jour à Concarneau, Douarnenez, Audierne et Saint-Guénolé (Camille Vallaux).

$1903: 1,60 \mathrm{~F}$ par jour à Moélan.

1905 : 1,50 F par jour à Douarnenez et à Concarneau.

1906 : 7 à 15 centimes de l'heure à Saint Guénolé, en grève pour avoir 20 centimes.

1908 : $150 \mathrm{~F}$ par mois, au minimum, pour les bigoudènes engagées dans les usines du bassin d'Arcachon.

1909 : 0,20 F de l'heure à l'usine Béziers des Sables d'Olonne; 0,15 F à Saint-Guénolé.

1910 : 0,25 F de l'heure à Concarneau, obtenu après une grève.

1911 : 0,20 F de l'heure à l'usine Lecointre de l'Herbaudière.

1913 : 0,17 F de l'heure à l'usine Basset de La Rochelle, à l'usine Palmer de Concarneau.

1914 : de 0,20 à $0,25 \mathrm{~F}$ de l'heure aux Sables d'Olonne, obtenu après une grève.

$1917: 0,30 \mathrm{~F}$ de l'heure aux Sables d'Olonne obtenu après une grève.

1917 : 0,25 F de l'heure à l'usine Basset de La Rochelle.

1918 : 0,40 F de l'heure à Audierne.

1920 : de 0,50 à $0,75 \mathrm{~F}$ de l'heure obtenu après une grève.

1922 : 0,60 F de l'heure à Douarnenez.

1923 : de 0,60 à $0,70 \mathrm{~F}$ de l'heure à Douarnenez, obtenu après une grève. 
1924 : 1,20 F de l'heure à Audierne;

$0,80 \mathrm{~F}$ de l'heure à Douarnenez mais obtiennent $1,20 \mathrm{~F}$ en décembre. de 1,00 à $1,20 \mathrm{~F}$ de l'heure à Concarneau.

$1925: 1,25 \mathrm{~F}$ de l'heure aux Sables d'Olonne

$1,00 \mathrm{~F}$ de l'heure à Audierne.

$1927: 1,35 \mathrm{~F}$ de l'heure en pays bigouden;

$1,50 \mathrm{~F}$ de l'heure à Douarnenez;

$1,25 \mathrm{~F}$ de l'heure à Croix-de-Vie demandé mais non obtenu.

1928 : de 1,25 à 1,50 F de l'heure aux Sables-d'Olonne

1930 : 1,75 F de l'heure à Pont-l'Abbé.

1935 : de 1,50 à 1,75 F de l'heure à l'usine Béziers de Camaret.

1936 : de 1,50 à 2,00 F de l'heure.

1937 : de 1,40 à 1,70 F de l'heure à Saint-Jean-de-Luz.

1939 : 2,35 F, grève à l'usine Petitjean d'Hennebont en novembre,

$1940: 2,60 \mathrm{~F}$ dans les usines du Guilvinec en novembre. 


\section{RÉSUMÉ}

En 1880, la France est le premier, et le seul, pays producteur au monde de conserves de sardines et de thons à l'huile distribuées sur la planète entière. 160 usines échelonnées de Trébeurden au bassin d'Arcachon préparent les poissons bleus, débarqués par 15000 à 20000 pêcheurs et travaillés par autant d'ouvrières. Ces femmes travaillent dans des conditions très difficiles, dans le vent et le froid l'hiver, dans des fours l'été; tous les jours où le poisson arrive et parfois 16 heures d'affilée pour que la précieuse sardine ne s'abîme pas. Les salaires versés sont les plus bas que toute la classe ouvrière perçoit alors en France. Et pourtant les mouvements de revendications des " filles de friteries " sont très rares et jamais organisés. Alors que le premier syndicat composé uniquement d'ouvrières est créé en 1874, pour assister aux premières associations syndicales féminines dans les conserveries de poisson, il faut attendre 1905 où, dans les deux plus grandes cités sardinières du pays, Douarnenez et Concarneau, s'ébauche une organisation structurée pour la défense de leurs intérêts, mais organisation éphémère dans un monde où le syndicalisme reste le privilège des hommes.

\section{ABSTRACT}

In 1880, France was the first and only country in the world producing sardines and tunny fish preserved in oil and distributed all over the world. 160 factories - from Trebeurden to the Arcachon Basin - prepared the blue fish landed by some 15,000 to 20,000 fishermen and processed by as many working girls. These women worked in very difficult conditions, in the wind and cold in winter, in ovens in summer; on all the days when the fish was coming, and sometimes 16 hours on end, so that the precious pilchard did not go bad. The salaries were the lowest paid in those days in France to the working class. However, industrial action by the "fish-girls" was of rare occurrence, and never organized. Though the first union composed of women only was founded in 1874, it was not until 1905 that one saw the first women's union in the fish-preserving factories. Then, in the two most important "sardine cities" in the country-Douarnenez and concarneau - was started a structured organization to defend their interests. But it was an ephemeral organization, in a world where syndicalism remained the privilege of men. 